\title{
Parallel real-world LU decomposition: Gauss vs. Crout algorithm
}

https://doi.org/10.1515/comp-2018-0020

Received July 7, 2018; accepted December 21, 2018

\begin{abstract}
This paper presents numerical experiments with assorted versions of parallel LU matrix decomposition algorithms (Gauss and Crout algorithm). The tests have been carried out on the hardware platform with fourcore Skylake processor featuring hyperthreading technology doubling virtually core number. Parallelization algorithms have been implemented with the aid of classic POSIX threads library. Experiments have shown that basic 4-thread acceleration of all parallel implementations is almost equal to the number of threads/processors. Both algorithms are worth considering in real-world applications (Florida University collection). Gauss algorithm is a better performer, with respect to timing, in the case of matrices with lower density of nonzeros, as opposed to higher density matrices. The latter are processed more efficiently with the aid of Crout algorithm implementation.
\end{abstract}

Keywords: parallel LU decomposition, Gauss decomposition, Crout decomposition, POSIX threads

\section{Introduction}

The problem of LU decomposition, even in parallel version, is not new. However little practical work on Gauss and Crout algorithms direct comparison has been carried out. First of all, most papers investigate separately Gauss algorithm and alternatively Crout algorithm, but not both of them. Moreover, almost all general research is based on synthetically generated matrix examples [1-4], i.e., on matrices with similar sparsity structure. Such approach may reveal the dependence of processing time and matrix size only. This paper presents comparison of parallel Gauss and Crout LU decomposition algorithms, based on real-world matrices examples. Both algorithms are arithmetically equivalent and the only difference is the or-

^Corresponding Author: Marek Stabrowski: Lublin University of Economics and Innovation, Poland; E-mail: marek.2491@gmail.com der/sequence of arithmetic operations. It is quite obvious that ordering of arithmetic operations may influence accuracy and stability of algorithm implementation. Less obvious is the impact of other factors including communication with cache memory, matrix sparsity structure (different in different real-world matrices) and compiler dependent optimizations. These latter factors may lead to spectacular differences in real processing time. Current research uses selected sparse matrices from Florida University collection. Real-world matrices are usually sparse. Matrix sparsity reflects the fact, that in complex systems individual components interact or are connected with limited number (not with all) of other system components. Sparse matrices may be processed using specialized sparse-oriented software [5-7], conserving memory space, or simpler dense matrix oriented software. LU decomposition, especially in pivoted version, leads in most cases to intensive fill-in of nonzeros. This phenomenon offsets the advantages of sparse-oriented software. Therefore in this research, sparse matrices are converted into dense form before decomposition. This conversion is carried out through insertion of nonzero elements, stored in compact HB format in Florida University collection, into corresponding locations in full square matrix/array. Next problem arises if the software selection is considered. Many renowned software packages either lack the option of alternative LU decomposition algorithms or, still worse, cannot guarantee sufficient numerical stability. The latter phenomenon may be observed, among others, in the case of Bayer matrices supplied by F. Grund. Therefore parallel implementation of Gauss and Crout LU decomposition has been developed specifically for the purposes of current research.

\section{Parallel Crout LU decomposition}

Basic serial implementation of Crout algorithm is presented in following pseudo-code listing. LU decomposition is performed in nuEqs steps (outer for loop), where $n u E q s$ is the number of equations/rows. In every $i$-th step the computations along L-shaped (turned clockwise by 90 
deg) front are carried out in two stages. Frontal column elements are computed in the first stage. Frontal row elements are computed in the second stage.

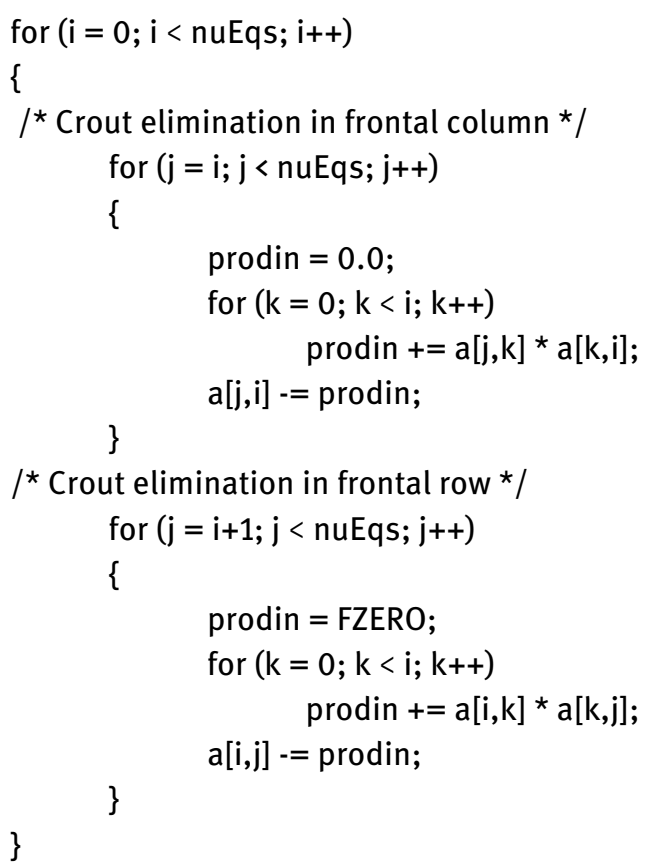

Pseudo-code of parallel version is shown below. Computations are distributed among several threads, separately in frontal column and in frontal row. Frontal column spans the rows starting with $i$-th row and ending with nuEqs. This set of rows is divided into nThreads (i.e., number of threads) segments with boundary indexes stored in rowLimits array. Individual thread processes appropriate segment of frontal column. After completion of column processing, the threads are joined. Frontal row is treated in similar manner. Division into segments uses the same boundary array. New set of parallel threads is forked and joined, after number crunching.

for $(\mathrm{i}=0 ; \mathrm{i}<$ nuEqs; $\mathrm{i}++)$

$\left\{/^{*}\right.$ Divide i...nuEqs into $\mathrm{nThreads}$ row blocks and store these block limits in rowLimits array*

$1{ }^{*}$ Crout elimination in frontal column * for (thread $=0$; thread $<$ nThreads; thread ++ )

$\left\{{ }^{*}\right.$ In real app forking of nThreads threads * for $(j=$ rowLimits[thread -1$] ; j<$ rowLimits[thread]; j++)

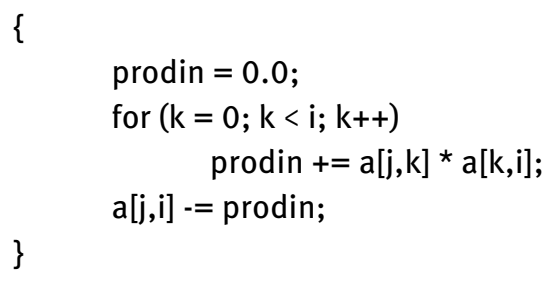

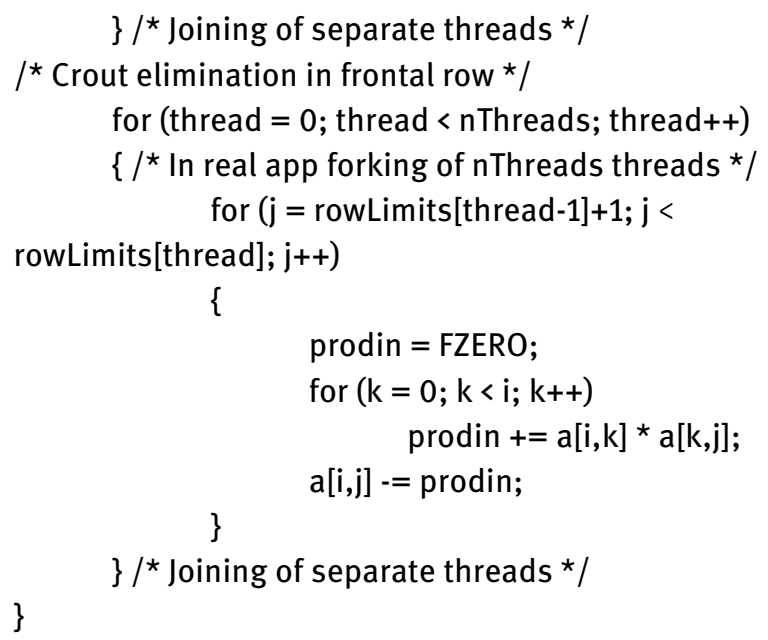

Basic data structures used in parallel implementation of Crout algorithm are presented graphically in Figure 1. This figure shows L-shaped computational front, the data involved in single decomposition step and basic division into parallel threads. It will be useful in further course in estimation of communication with cache memory.

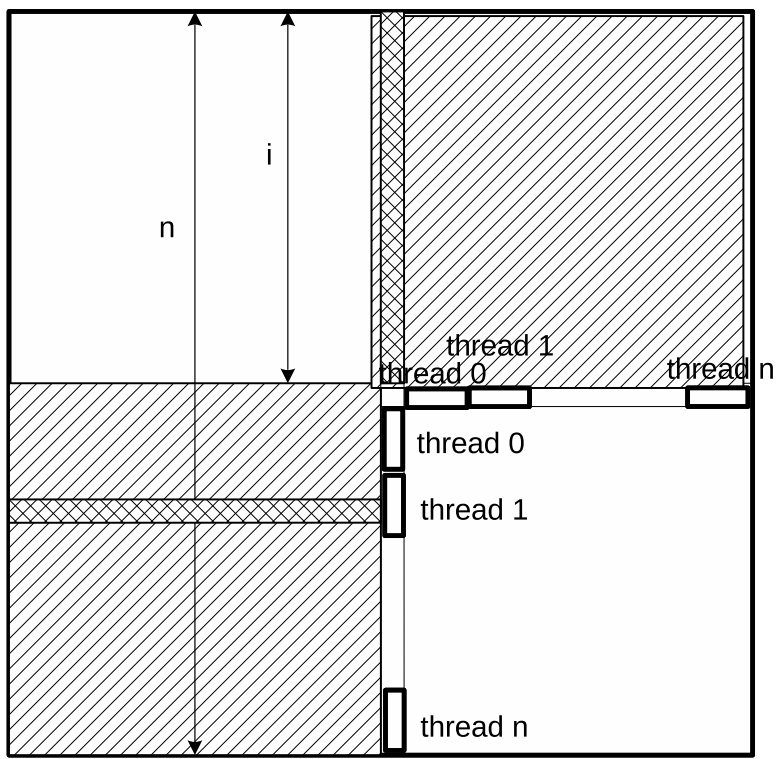

Figure 1: Basic data structures in parallel Crout LU decomposition.

It is quite obvious, that in order to get optimum efficiency, the number of threads should be equal to the number of parallel cores/processors. Computations in Crout algorithm involve rather large number of already determined matrix elements. Pivoting in Crout algorithm is performed, usually in rather straightforward way, without resorting to parallelization. Matrix elements inside L-shaped front 
(lower right submatrix) are modified only gradually and therefore scaled equilibration is accepted as wholly sufficient.

\section{Parallel Gauss LU decomposition}

Core operations of Gauss LU decomposition are composed of three nested loops. Almost all parallel implementations prefer $i j k$ arrangement of these loops $[1,6]$. Such approach has been adopted in current research. Pseudo-code of serial version is presented below.

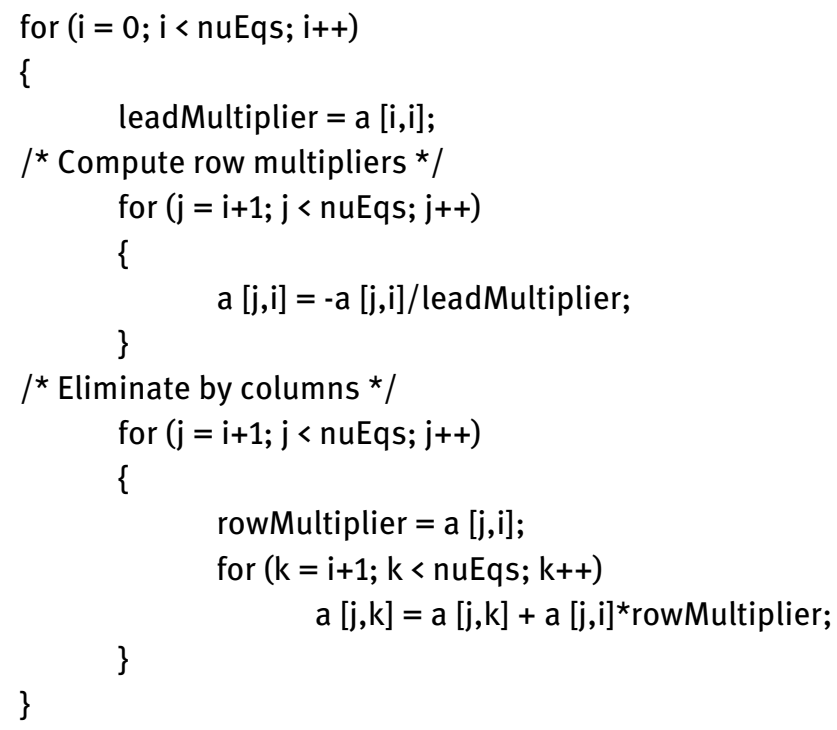

Important key decision in multi-threaded implementation is related to granularity of parallel segmentation. For low or mid-range parallel hardware platforms, strip structure exploits sufficiently multiprocessing capabilities $[5,6]$. It has been adopted in implementation presented here. Division of macrorows into finer blocks (grains) may be more adequate on massive multicore hardware.

Pseudo-code of parallel version is shown below. Computations are distributed among several threads, with individual threads processing a block of rows. Boundary indexes of the row blocks are stored in rowLimits array. New set of parallel threads is forked and joined, after single LU decomposition step with appropriate modification of block boundary indexes.

for $(i=0 ; i<$ nuEqs; $i++)$

\{

/* Divide i...nuEqs into nThreads row blocks and store these block limits in rowlimits array ${ }^{*}$ for (thread $=0$; thread < nThreads; thread ++ )

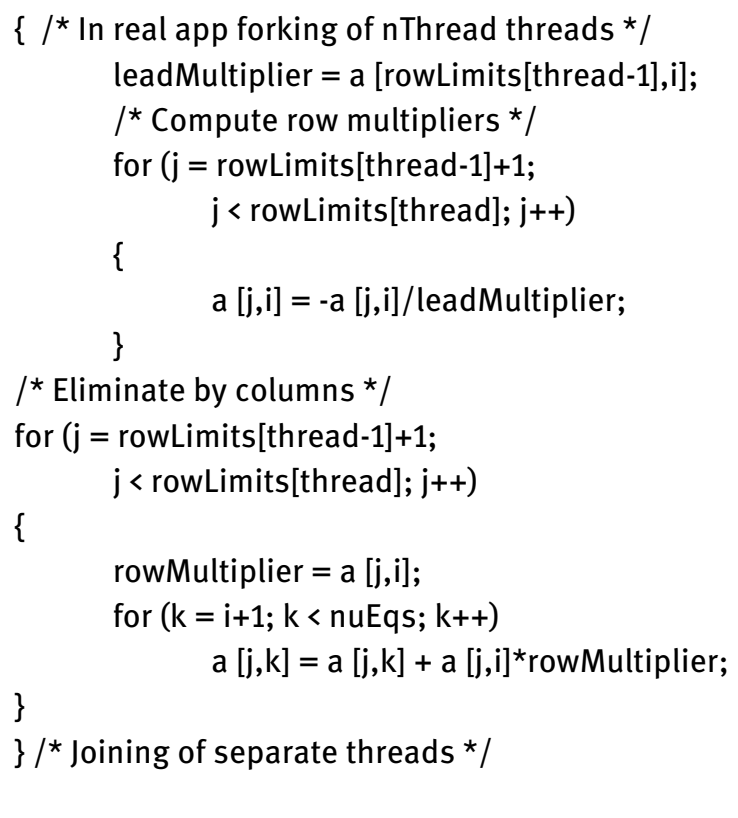

Basic data structures used in parallel implementation of Gauss algorithm are presented graphically in Figure 2. This figure shows division into row blocks processed in individual parallel threads. Even most cursory comparison with similar figure for Crout algorithm (Figure 1) shows that Gauss algorithm is much more economical with respect to data usage. In further course quantitative estimation of communication with cache memory will be presented.

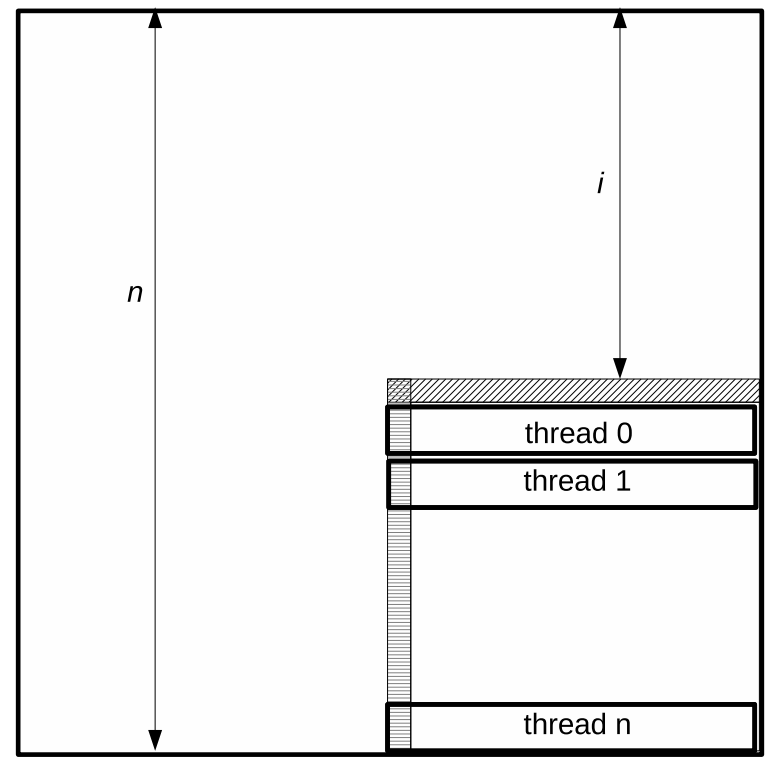

Figure 2: Basic data structures in parallel Gauss LU decomposition. 
In order to secure sufficient numerical stability, partial pivoting with implied scaling has been used in actual algorithm implementation. It can be easily observed in the pseudo-code quoted that modifications in lower right submatrix are carried out in every step of LU decomposition. Therefore the details and whole general picture of pivoting decisions differs from the simple strategy adequate for Crout algorithm. Pivoting decision in $i$-th decomposition step is based on the normalized value of leading element of current $j$-th row, i.e.

$$
a_{j i} / \sum_{k=i+1}^{n}\left|\left(a_{j k}\right)\right|
$$

This normalization of pivoting elements engages relatively large processing power and must be also parallelized. Therefore single step of parallel Gauss algorithm is composed of parallel pivoting section, actual pivoting interchange (or not) and parallel Gauss elimination. The data structures in pivoting section are identical with the ones in elimination section. Individual threads in pivoting section determine local (thread-specific) pivot candidates. Joining of pivoting threads determines global and final pivot through simple selection.

\section{Hardware and software platform}

Numerical experiments have been performed on a computer with Skylake 6700HQ processor running Linux (Fedora 28) operating system. Skylake processor features 4 physical cores with additional 4 virtual cores resulting from hyperthreading. There is a bug in Skylake original microcode, which impairs execution of tight 64-instructions loops (several reports in Internet media, started by LinuxDebian community). However this malicious behaviour was absent in the software used. The structure and size of cache memory is another important architectural feature of Skylake processor. Cache memory impacts processor performance due to the gap between processor speed (high) and main memory speed (low). Cache memory is formed by four sets of three-level structures. Every set is directly connected to single physical core. In effect, physical and virtual (hyperthreading) cores share the same structure of cache memory. Sharing of cache memory may result in conflicts and collisions. Such structure is composed of level L1 memory (64 kB/core), level L2 memory (256 $\mathrm{kB} /$ core) and level L3 memory (2 MB/core). The software, used in curent tests, was developed in $\mathrm{C} / \mathrm{C}++$ language and compiled with GNU compiler version 8.1.1-5. Parallelization was performed through thread generation with the aid of the POSIX threads library [8]. It is relatively low-level library having reputation of highest timing efficiency.

\section{Real-world test matrices}

Test matrices were selected from huge Florida University collection. They were generated in real-world applications. Basic characteristics of these matrices are presented in tables 1 and 2. Some of the test matrices describe chemical engineering processes (Gru30, LHR04C, HYDR1), dielectric waveguides (DW4096), population migration (PSMIGR1), Markov chains (RW5151), The size/dimension of these matrices spans the range of $3000+$ to $8000+$. This range is adequate for the processing power of the hardware used in the current research. All these matrices are sparse, but for the purposes of current research they have been converted into the dense ones. Initial density of nonzeros (the ratio of nonzeros number to all elements) is well below $0.5 \%$ with the exception of PSMIGR1 matrix, which reaches a density of 5.5\%. LU decomposition results in fill-in of zeros. Fill-in is the ratio of nonzeros after LU decomposition and before. This fill-in is larger in the case of Crout method. Typical values of fill-in range from 5 to 100 . Sparse implementations of $L U$ decomposition algorithms are less suited to cope with such fill-in. However the ruling in favour of sparse or dense implementations is beyond the scope of the current research. Basic processing time of sequential (single thread) implementation of both algorithms, shown in table 1 , is in the range from $14+$ seconds, up to $5500+$ seconds.

Table 1: Sample real-world matrices used in current tests.

\begin{tabular}{lcccrr}
\hline & $\begin{array}{c}\text { No. } \\
\text { of } \\
\text { equa- } \\
\text { tions }\end{array}$ & $\begin{array}{c}\text { Initial } \\
\text { non- } \\
\text { zeros } \\
{[\%]}\end{array}$ & $\begin{array}{c}\text { Non- } \\
\text { zeros } \\
\text { after } \\
\text { Gauss } \\
{[\%]}\end{array}$ & $\begin{array}{c}\text { Time } \\
\text { se- } \\
\text { quen- } \\
\text { tial } \\
\text { Gauss } \\
\text { [sec] }\end{array}$ & $\begin{array}{c}\text { Time } \\
\text { se- } \\
\text { quen- } \\
\text { tial } \\
\text { Crout } \\
\text { [sec] }\end{array}$ \\
\hline Gru30 & 3268 & 0.194 & 0.944 & 14.6 & 43.8 \\
DW4096 & 8192 & 0.062 & 5.425 & 398.0 & 5587.8 \\
HYDR1 & 5308 & 0.080 & 0.331 & 63.2 & 310.8 \\
LHR04C & 4101 & 0.492 & 19.425 & 49.3 & 134.1 \\
PSMIGR1 & 3140 & 5.509 & 94.943 & 106.9 & 35.8 \\
RW5151 & 5151 & 0.076 & 1.298 & 56.1 & 314.3 \\
\hline
\end{tabular}




\section{Experimental comparison of Gauss and Crout LU decomposition}

Computational efficiency of multithreaded algorithm depends primarily on the possibility of selecting and distributing orthogonal computational operations between individual threads. Such orthogonal sets of operations have been shown in pseudo-code in sections 2 and 3 . Second factor influencing this efficiency is the overhead of thread structures (boundaries of thread arrays, number of arrays) in both algorithms. It can be easily observed (see pseudo-code) that Gauss algorithm uses only $n$ thread structures in single decomposition step in contrast to $2 n$ thread structures of Crout algorithm. Also other factors can influence computational efficiency [9]. Generally, it is expected that parallel implementation of an algorithm, which may be effectively parallelized, should shorten the processing time by the factor equal to the number of threads. This expectations are fulfilled only partially on the software and hardware platform used in the current research. Lets start with multithreaded Crout algorithm (Figure 3).

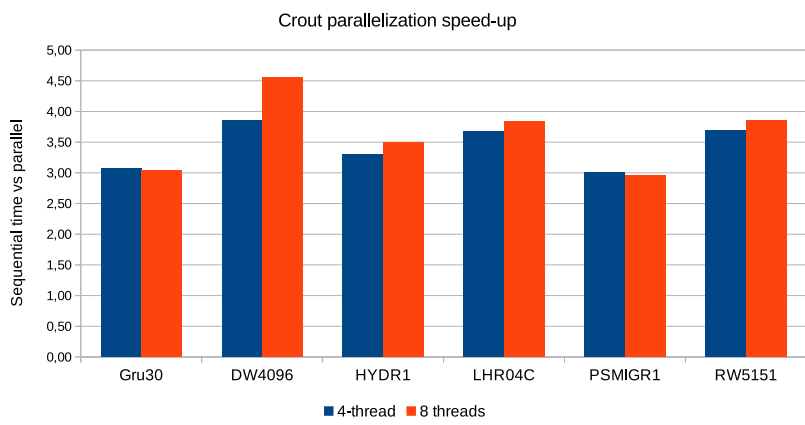

Figure 3: Speedup of parallel Crout LU decomposition vs. sequential one

It follows from Figure 3, that parallel Crout algorithm in 4-thread version speeds-up LU decomposition by the factor of 3.5 up to almost 4. This is good confirmation of parallelization potential of Crout algorithm. However, the 8-thread version of Crout algorithm fails to fulfill naive expectations of further speedup. Almost in all cases, with the exception of DW4096 matrix, processing time of 8-thread version is approximately equal to the time of 4-thread version. Only in the case of DW4096 matrix 8-thread version outperforms 4-thread version by $30 \%$. This failure of 8 thread version follows from the fact, that Skylake proces- sor is physically a 4-core structure and 8-core operation is implemented through hyperthreading.

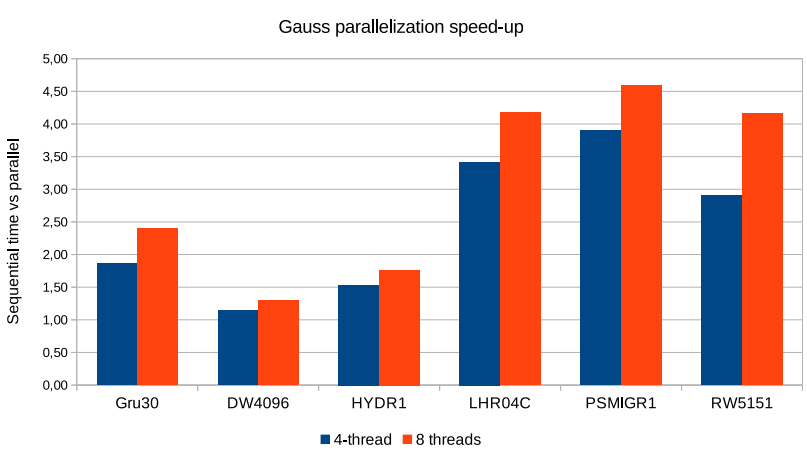

Figure 4: Speedup of parallel Gauss LU decomposition vs. sequential one.

Speedup of Gauss algorithm (Figure 4) due to parallel implementation on Skylake platform differs slightly from Crout algorithm. It can be observed that 4-thread version is also 4 times faster than sequential version but some interesting exceptions may be observed. In the case of LU decomposition of DW4096 matrix the speedup is hard to observe and for HYDR1 matrix does not reach the value of 3. For other matrices used in this research the speedup of 4-thread version is around 4 or even higher. The 8-thread version offers in most cases higher speedup than similar version of Crout algorithm. In the case of RW5151 matrix the 8-thread version is 2 times faster than 4-thread version. In other cases the speedup is markedly lower down to negligible value for HYDR1 matrix. Thus hyperthreading fails to implement efficiently parallelization of LU decomposition similarly, as in the case of Crout algorithm.

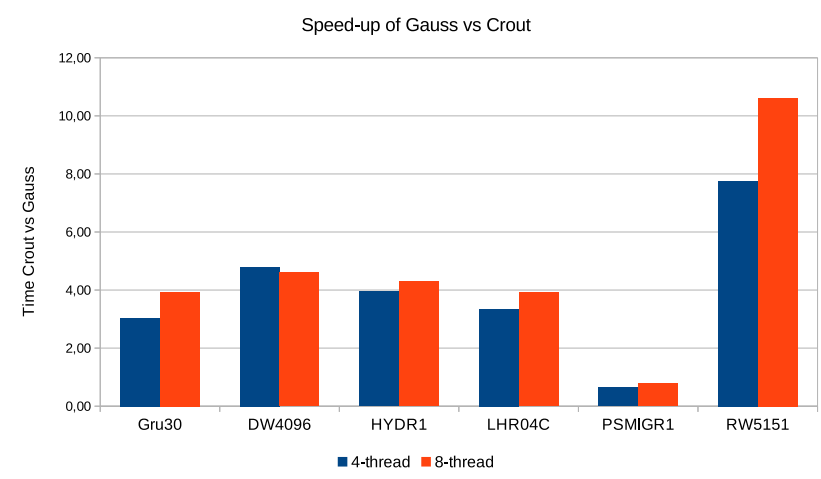

Figure 5: Comparison of parallel Crout LU decomposition algorithm vs. parallel Gauss. 
Comparison of parallel Gauss LU decomposition with Crout algorithm (Figure 5) also leads to interesting observations. Gauss 4-threaded algorithm is in most cases 3 to 5 times faster than analogous Crout algorithm. In the case of 8-threaded versions, Gauss algorithm speedup is similar with the exception of RW5151 matrix, where it is around the factor of 8. It is easy to conclude that Gauss algorithm, despite arithmetic equivalence, is better performer than Crout algorithm. However, there is interesting exception with the PSMIGR1 test matrix. In the case of this matrix parallel Crout algorithm, in both versions tested, offers processing time shorter by the factor of 2 .

\section{Selected factors influencing computational efficiency}

Experimental results of processing times for selected realworld matrices have been presented in previous section. It has been already reminded, that Gauss and Crout algorithm differ only with respect to arithmetic operations order. The number of arithmetic operations is identical. Therefore explanation of spectacular differences of computational efficiency (Figure 5) must be based on more subtle factors.

Various software and hardware optimizations may contribute to computational efficiency (or processing time) of algorithm implementation for real-world matrices. It has been proven in the current research that these optimizations depend on the sparsity structure of the matrices. It follows from Table 1 and Figure 5, that PSMIGR1 matrix stands apart from the rest of the matrices. Even in it's original form it is quite dense ( $5 \%$ of nonzeros), and after LU decomposition the density reaches $100 \%$. Crout indexing (fixed point arithmetic) is more compact, and therefore may be better optimized by the compiler. Due to hardware advances in quite remote past, floating point operations timing does not differ from fixed ones timing, and therefore floating point operations are no longer a decisive factor in assessment of algorithm efficiency. Therefore Crout algorithm for this matrix group shows far better performance than Gauss algorithm. At present precision assessment of hardware and software optimizations on both algorithms efficiency may be performed only experimentally.

Communication with cache memory is another important factor influencing computational efficiency. Here some form of quantitative analysis may be performed. In the case of LU decomposition algorithms, the system matrix is the largest data structure. In the case of
LHR04C equation system, it is composed of approximately $16.5 \times 10^{6}$ floating point numbers (number of equations is 4101). The Skylake processor used as the test platform was equipped with $8 \mathrm{MB}$ of L3 cache memory. This memory is sufficient for storing $10^{6}$ double precision numbers, i.e. about $6 \%$ of whole system matrices. It is evident, that fast and efficient algorithm should exploit fully the matrix chunk currently in cache memory, proceed to the next chunk, without returning (in ideal case) to previous contents of the cache. System matrix on the level of assembly language or on the conventional machine level, is onedimensional linear structure with row-oriented ordering (C/C++ language). Crout algorithm during specific $i$-th decomposition step uses only coefficient matrix fragments adjacent to L-shaped computational front. Due to internal one-dimensional matrix representation, whole matrix must be accessed (and transmitted through the cache memory) in every decomposition step. It can be observed that the elements from first matrix row, as well as the elements from last row of the matrix are used. Thus in $i$-th step of Crout algorithm, the number $n_{i}$ of matrix elements transmitted through the cache memory is equal to

$$
n_{i}=n^{2}
$$

where: $n$ - number of equations (matrix dimension). This number does not depend on the number of decomposition step. Therefore, overall number $n_{C}$ of matrix elements transmitted through the cache memory in Crout algorithm is equal to

$$
n_{C}=n^{3}
$$

The situation is different in the case of Gauss algorithm. In the $i$-th decomposition step only lower right rectangular part of the matrix (see Figure 2) is active. The size of this rectangle is equal to

$$
n_{i}=(n-i) n
$$

It is necessary to note, that only a square submatrix, containing $(n-1) 2$ elements must be traversed in $i$-th step. One-dimensional linear representation of coefficient matrix forces transmission of entire lower right rectangle submatrix. And the total number of transmitted elements during LU decomposition using Gauss algorithm is approximately equal to

$$
n_{G}=n^{3}
$$

This estimation is a bit pessimistic, as in final steps of LU decomposition, transmitting new elements into cache memory is not necessary. For example in smallest equation system analysed, one row of double precision coefficients occupies $11 \mathrm{kB}$ of memory. Last 80 steps of decomposition use the coefficients already present in cache memory 
of $1 \mathrm{MB}$. Direct comparison of cache transmission workload for both algorithms (equations (3) and (5)) leads at first sight to the conclusion that Gauss algorithm is 3 times faster than Crout algorithm. This is the relative "data access locality" comparing Gauss vs. Crout algorithm. However, cache refilling is only some part of total computational work. Moreover, Crout algorithm performs about 2/3 of Gauss fixed point operations. Indexing work in Crout algorithm, due to compact formulas, consumes less processor time. These factors reduce the advantages of Gauss algorithm data locality. This analysis explains spectacularly better efficiency of Gauss algorithm presented in Figure 5.

Table 2: Profiling of basic Crout algorithm.

\begin{tabular}{lcccc}
\hline & $\begin{array}{c}\text { Instru- } \\
\text { ctions }\end{array}$ & Data & $\begin{array}{c}\text { L1 } \\
\text { misses } \\
{[\%]}\end{array}$ & $\begin{array}{c}\text { L3 } \\
\text { misses } \\
{[\%]}\end{array}$ \\
\hline Gru30 & $0.49 \mathrm{e} 12$ & $0.25 \mathrm{e} 12$ & 4.8 & 0.6 \\
DW4096 & $7.71 \mathrm{e} 12$ & $4.04 \mathrm{e} 12$ & 5.0 & 4.8 \\
HYDR1 & $2.10 \mathrm{e} 12$ & $1.10 \mathrm{e} 12$ & 5.0 & 0.6 \\
LHR04C & $0.97 \mathrm{e} 12$ & $0.51 \mathrm{e} 12$ & 4.9 & 0.6 \\
PSMIGR1 & $0.44 \mathrm{e} 12$ & $0.23 \mathrm{e} 12$ & 4.8 & 0.6 \\
RW5151 & $1.92 \mathrm{e} 12$ & $1.00 \mathrm{e} 12$ & 5.0 & 0.6 \\
\hline
\end{tabular}

Table 3: Profiling of basic Gauss algorithms.

\begin{tabular}{lcccc}
\hline & $\begin{array}{c}\text { Instru- } \\
\text { ctions }\end{array}$ & Data & $\begin{array}{c}\text { L1 } \\
\text { misses } \\
{[\%]}\end{array}$ & $\begin{array}{c}\text { L3 } \\
\text { misses } \\
{[\%]}\end{array}$ \\
\hline Gru30 & $0.31 \mathrm{e} 12$ & $0.18 \mathrm{e} 12$ & 0.9 & 0.8 \\
DW4096 & $4.98 \mathrm{e} 12$ & $2.86 \mathrm{e} 12$ & 1.1 & 1.1 \\
HYDR1 & $1.31 \mathrm{e} 12$ & $0.75 \mathrm{e} 12$ & 0.9 & 0.8 \\
LHR04C & $0.69 \mathrm{e} 12$ & $0.39 \mathrm{e} 12$ & 1.7 & 0.8 \\
PSMIGR1 & $0.70 \mathrm{e} 12$ & $0.38 \mathrm{e} 12$ & 5.3 & 0.6 \\
RW5151 & $1.20 \mathrm{e} 12$ & $0.70 \mathrm{e} 12$ & 0.9 & 0.8 \\
\hline
\end{tabular}

More insight may be gained through profiling of basic implementations of both algorithms. The results of such operation are presented in Table 2 and Table 3. For all tested matrices, with notable exception of PSMIGR1 matrix, highest level (L1) cache misses are significantly lower for Gauss algorithm, ranging from $0.9 \%$ up to $1.7 \%$. In contrast this ratio for Crout algorithm falls into the range of $4.8 \%$ up to 5.0. Also instruction count and data count is lower by approximately (Table 2, Table 3) $40 \%$. Therefore profiling tests prove superiority of Gauss algorithm vs.
Crout algorithm. However PSMIGR1 matrix, differing with respect to sparsity pattern, shows also different profiling properties. Cache misses are slightly below corresponding results for Gauss algorithm. More spectacular is the difference of executed instructions count and accessed data; this count is lower by about $40 \%$ in favour of Crout algorithm. It proves the superiority of Crout algorithm for such type of matrices.

One may expect that running more parallel threads, i.e., eight in place of four, appropriately speeds up the processing. Skylake processor offers nominally eight processors and therefore splitting the computations into eight threads is, at least theoretically, optimum choice. However the results shown in Figure 4 and Figure 5 do not fulfill this expectation. The 8-thread versions of both algorithms do not offer significant timing advantages and in the case of Gauss algorithm are even slower than 4-thread versions. This phenomenon can be explained with the fact, that there are only four physical processors in Skylake chip and additional four virtual processors are resulting from hyperthreading. Cache memory, critical for multithreaded operations, is directly connected to physical processors and therefore there are four segments of cache memory. In hyperthreading mode these cache memory segments are shared by physical processor and a virtual one. There may arise conflicts and collisions during intensive accessing of this memory by physical processor and virtual one. It is the explanation of 8-threaded versions poor performance observed in Figure 3 and Figure 4.

\section{Conclusions}

Reported research results lead to the conclusion that computational efficiency (or timing) of parallel LU decomposition algorithms depends on the matrix characteristic features. They include sparsity pattern of the matrix, i.e., the ratio of nonzeros and total number of elements. Both tested algorithms, despite identical basic (i.e., floating point) arithmetic operation count, show different performance in the case of real-world matrices. In most cases Gauss algorithm is a better performer than Crout algorithm. This phenomenon is attributable to distinctly lower cache memory usage. However Crout algorithm is a better choice in the case of dense matrices. Hyperthreading technology, in the case of the hardware used, failed to fulfil the expectations. Only in the case of Gauss algorithm meaningful speedup of 8-thread version was observed. This limitation does not depend on the known hardware bug of 
Skylake processor, but rather on the cache sharing between physical and virtual cores.

\section{References}

[1] Higham N. J., Gaussian elimination (Advanced Review), In: WIREs Computational Statistics, 2011, 3(3), 230-238

[2] Mayer J., A multilevel Crout ILU preconditioner with pivoting and row permutation, Numerical Linear Algebra with Applications, 2007, 14(10), 771-789

[3] Sibai F. N., Performance modeling and analysis of parallel Gaussian elimination on multi-core computers, Journal of King Saud University - Computer and Information Sciences, 2014, 26, 4154

[4] Zhang L., Sun Y., Ma J., A parallel Crout algorithm based on TBB, In: Proceedings of the 2nd International Conference on Software Engineering and Service Science, IEEE, 2011, 239-242
[5] Demmel J. W., Eisenstat S. C., Gilbert J., Li X., Liu J. W. H., Technical Report UCB//CSD-95-883, Computer Science Division, U.C. Berkeley, 1995

[6] Donfack S., Dongarra J., Faverge M., Gates M., Kurzak J., Łuszczek P., Yamazaki I., On algorithmic variants of parallel Gaussian elimination: comparison of implementations in terms of performance and numerical properties, Research Report, 2013, https://hal.inria.fr/hal-00867837

[7] Huckle T., Parallel numerics, Technische Universität München, Institut für Informatik, 2006

[8] Butenhof D. R., Programming with POSIX Threads, Addison Wesley, Boston, 1997

[9] Kaya D., Wright K., Parallel algorithms for LU decomposition on a shared memory multiprocessor, Applied Mathematics and Computation, 2005, 163(1), 179-191 